Background After 12 years of activity the integrated sexual health service in Glasgow, Scotland begun to plan for the future. The service's evolution from previously separate units improved access to sexual health care, however, maintaining and growing innovative services had become challenging, with restricted resources and staffing, and complex client demand. The service now has 20 sites, 250 clinical, support and management staff and sees 100, 000 clients annually.

When its manager returned from another role, the service started to consider its future delivery model. This paper sets out the methodology based around appreciative inquiry and staff engagement, and includes feedback from staff about its impact on them and the organisation.

Methods The process began in June 2012 with a Planning Day for senior staff which agreed a timetable, identified staff and others to involve; scoped essential areas such as public engagement, consultation, communication, and evidence like population projections and service data, and agreed a shared vision. Personal narratives and goals were shared to shape the vision and to acknowledge the past. A multi-disciplinary Steering Group was then established to participate in planning and decision-making, with a Staff Council arising through staff interest - both now with significant roles. Regular staff communications and large engagement events have also helped strengthen plans and organisational structures.

Results The service's future vision for developing sexual health services acknowledges its history and proposes clear objectives that re-iterate a commitment to addressing inequalities and to proportionate universalism. This has been achieved in an inclusive manner that has empowered staff across the organisation and included their views and ideas.

Summary This paper describes a systematic approach to engaging with a large and diverse staff group through appreciative inquiry and other methods to improve the sexual health services in the Glasgow area of Scotland.

\section{P6.002 EFFICACY OF CHLAMYDIA CONTROL PROGRAMMIS: OPTIMAL COMBINATIONS OF PARTNER NOTIFICATION AND SCREENING IN A PAIR APPROXIMATION MODEL}

doi:10.1136/sextrans-2013-051184.1156

1J Clarke, ${ }^{2} \mathrm{~J}$ K White, ${ }^{2} \mathbf{K}$ M Turner. 'University of Bath, Bath, UK; ${ }^{2}$ University of Bristol, Bristol, UK

Background Chlamydia control in England is based on management of positives and their partners (partner notification) and on identifying asymptomatic infections in the community (screening).

Aims To describe the relative contribution of screening and partner notification to control of chlamydia at different stages in the epidemic and evaluate optimal resource allocation to each control.

Methods Using pair approximation equations we investigate the efficacy of control programmes for chlamydia on short time scales that are relevant to policy makers. We estimate prevalence, incidence, and positivity in those screened and in their partners. We combine these measures with a costing tool to estimate the economic impact of different public health strategies.

Results Increasing screening coverage significantly increases the annual programme costs whereas an increase in tracing efficiency initially increases annual costs but over time reduces costs below baseline, with tracing accounting for around $10 \%$ of intervention costs. We found that partner positivity is insensitive to changes in population prevalence due to screening, remaining at around $33 \%$.

Conclusions Maintaining efficacy of partner notification is costeffective at all stages of the epidemic (stable/declining prevalence) but becomes increasingly cost-effective as control measures reduce the population prevalence.

\section{P6.003 GEOGRAPHIC MAPPING OF FEMALE SEX WORKERS AND VENUE PROFILING IN URBAN AND RURAL DISTRICTS OF ZIGONG, SICHUAN, CHINA}

doi:10.1136/sextrans-2013-051184.1157

'J F Blanchard, ${ }^{2} \mathrm{~J}$ Zhang, ${ }^{2} \mathrm{H}$ Zhou, ${ }^{2} Y$ Yang, ${ }^{3} Y \mathrm{Xie},{ }^{3} \mathrm{O} \mathrm{Li},{ }^{1} \mathrm{~F}$ Emmanuel, ${ }^{2} \mathrm{X} \mathrm{Ma},{ }^{1} \mathrm{R}$ Lorway, 'B Yu. 'University of Manitoba, Winnipeg, MB, Canada; ${ }^{2}$ Sichuan University, Chengdu, China; ${ }^{3}$ Zigong Center for Disease Prevention and Control, Zigong, China

Introduction The purpose of this study is to geographically enumerate the population size of female sex workers (FSWs) and venue profiling of key venues, and to reveal the key elements which are related to the high risk sexual behaviours among FSWs in urban and rural areas of Zigong city in Sichuan, China.

Methods Geographic mapping data were collected through systematically identifying hidden key venues in the rural and urban districts of Zigong. Venue profiling data were collected by interviewing key informants (KI) about the details of sex work operation, such as type of venue, duration of operation, operation days and time, peak days and time, services provided at each venue, number of clients on an average day and a peak day. To avoid social desirability bias of face-to-face interview, Polling Booth Survey was used to gather high risk sexual behaviours among FSWs $(\mathrm{N}=60)$.

Results A total of 324 key venues were mapped in Zigong. The key venues are massage parlours (108), teahouses (74) and small hotels (45), which accounting for $33.3 \%, 22.8 \%$ and $13.9 \%$ of total venues mapped. 112 venue KIs were interviewed and confirmed a total of 378 FSWs working in those 112 venues. The average number of FSWs per venue is 4 . The age of the majority (80.4\%) of FSWs was around 20 to 40 years old. The total estimated number of FSWs in Zigong is 1296 . The sexual behaviours and operation patterns of key venues in urban and rural areas are different. Not consistent condom use, STI symptoms, and drug use are some typical high risk behaviours.

Discussion and Conclusion Sex work industry is emerged in general social life in urban and rural China. The scope and operation of sex industry pose a special challenge to public health intervention programmes

\section{P6.004 THE PREVALENCE OF SEXUALIY TRANSMITTED INFECTIONS AMONG CLIENTS OF FEMALE SEX WORKERS IN KARNATAKA, SOUTH INDIA: AN ANALYSIS BY PLACES OF SOLICITATION OF SEX WORKERS}

doi:10.1136/sextrans-2013-051184.1158

${ }^{1} \mathbf{S}$ Y Shaw, ${ }^{2} \mathrm{P}$ Bhattarcharjee, ${ }^{2} \mathrm{~S}$ Isac, ${ }^{3} \mathrm{~K} N$ Deering, ${ }^{2} \mathrm{~B} M$ Ramesh, ${ }^{2} \mathrm{R}$ Washington, ${ }^{1} S$ Moses, ' J F Blanchard. 'University of Manitoba, Winnipeg, MB, Canada; ${ }^{2}$ Karnataka Health Promotion Trust, Bangalore, India; ${ }^{3}$ University of British Columbia, Vancouver, $B C$, Canada

Background Clients of female sex workers (FSWs) are an important bridging population for HIV/STI transmission. Designing client-specific intervention programming faces several challenges, as clients do not comprise one single identifiable group. This study sought to describe HIV/STI prevalence by places of solicitation, among clients in south India.

Methods Data were from two rounds of cross-sectional biological and behavioural surveys of FSW clients from Karnataka State, India. Where available, the prevalence of HIV, active syphilis, herpes simplex virus type 2 (HSV-2), chlamydia (CT) and gonorrhoea (GC) was examined. Separately for each survey round and infection, multivariable logistic regression models were used to examine differences in infection prevalence by solicitation site, adjusted for clients' district. Solicitation sites were categorised as public place, brothel, home and lodge. Sampling weights and survey methods were utilised in regression models. 
Results Total sample size was 2,331 and 2,282 in Rounds 1 and 2, respectively. In Round 1, syphilis (6.1\%), HIV (9.5\%) and HSV-2 $(31.3 \%)$ was highest among clients soliciting FSWs from brothels. CT/GC (6.6\%) was highest among clients of FSWs based in lodges. In Round 2, syphilis (3.2\%), HIV (6.9\%) and HSV-2 (20.5\%) were highest amongst clients soliciting in lodges, brothels and public places, respectively. Statistical significance was reached for HIV $(p=0.003)$ and CT/GC $(p=0.011)$ by site in Round 1 only. Differences by solicitation site in Round 1 remained significant when adjusted for district. Between rounds, prevalence decreased in all solicitation sites, with the exception of syphilis $(2.0 \%$ to $3.2 \%)$ and HIV $(0.6 \%$ to $1.1 \%)$ among clients frequenting lodges.

Conclusions Clients frequenting brothels had the highest HIV prevalence. Further study of the increasing prevalence of syphilis and HIV among clients frequenting lodges is warranted. Approaches focussing on where clients solicit FSWs, rather than identifying clients per se can help inform programming activities.

\section{P6.005 MULTIDISCIPLINARY APPROACH TO MANAGING A SYPHILIS OUTBREAK IN SOUTHEND-ON-SEA, ESSEX UK}

doi:10.1136/sextrans-2013-051184.1159

'K A Fernando, ${ }^{2} \mathrm{~S}$ Matthews, ${ }^{1} \mathrm{H}$ Jaleel, ${ }^{3} \mathrm{~S}$ Salimee. 'Department of Sexual Health \& HIV Medicine, Southend University Hospital NHS Foundation Trust, Essex, UK; ${ }^{2}$ Department of Public Health, Essex Primary Care Trust, Essex, UK; ${ }^{3}$ Essex Health Protection Unit, Health Protection Agency, Essex, UK

Background In 2012 the Essex Health Protection Unit of the Health Protection Agency(HPA) was alerted to a significant rise in syphilis in Southend. 27 cases were diagnosed in 2011 and 15 in the first half of 2012, compared to just 10 in 2010, indicating a greater than $50 \%$ rise. All were in men who have sex with men. In England as a whole, in 2011, 2915 syphilis cases were diagnosed, reflecting a less modest $10 \%$ rise compared to 2010 .

Importantly, Southend is a high prevalence UK area of HIV, with a population prevalence of 2.76 per 1000 . 6 of 27 individuals diagnosed with syphilis in Southend in 2011 were HIV-infected.

Aim A multidisciplinary incident management team (MDT) was established to investigate and establish measures to control the outbreak. The MDT includes members of the local health authority, public health department, HPA and genitourinary medicine(GUM) department. The MDT agreed to monitor GUM and diagnostic laboratory activity to inform testing and prevention strategies.

Interventions Local media press releases alerting the public of the syphilis outbreak were produced. Such information advised on sexual health promotion, irrespective of age or perceived risk. The Gay Essex Men's Support Group, Terrence Higgins Trust, and local contraception services were also alerted and encouraged to use existing networks to raise awareness.

Annual use of one of 6 pharmacy campaigns to raise STI awareness was implemented. Dissemination (verbally and writtenintranet) of information on STI trends and management was well received by local general practitioners.

Conclusions Firstly, local accurate reporting confirmed the increase in syphilis diagnoses, and enabled timely review and public health action. Secondly, good interagency and multidisciplinary working resulted in productive collaborative responses. And finally, the use of existing sexual health and MSM social/support networks enabled access to 'harder to reach' populations with regards to STI health promotion.

\section{P6.006 COUNTING THE PENNIES: RATIONAL PRESCRIBING WITHIN THE CONTEXT OF THE NATIONAL CHLAMYDIA SCREENING PROGRAMME}

doi:10.1136/sextrans-2013-051184.1160

'K M Turner, ${ }^{1} \mathrm{P}$ Horner, ${ }^{1} \mathrm{I}$ Vincent, ${ }^{2} \mathrm{E}$ Adams, ${ }^{1} \mathrm{~J}$ Macleod. ' University of Bristol, Bristol, UK: ${ }^{2}$ Aquarius Population Health, London, UK
Background In 2010/11 the National Chlamydia Screening Programme diagnosed over 90,000 cases of chlamydia in England. The current BASHH guidelines recommend either Azithromycin or doxycycline. Concerns have been raised questioning the efficacy of Azithromycin in routine practise.

Aims The aim of our study was to analyse whether current prescribing is in line with the criteria for rational prescribing and to estimate the cost and efficacy implications of a change in prescribing practise.

Methods We searched for published data on clinical effectiveness (both in randomised controlled trials and in practise), cost, compliance, universal use, acceptability, antimicrobial resistance and safety profiles.

We developed a simple costing tool to estimate the potential cost implications of prescribing practise and clinical effectiveness on the total treatment cost and the cost per infection treated.

Findings Until 2012, Azithromycin cost £9.65 per 4-tab 250mg pack in comparison with $£ 1.70$ per 28 capsule $50 \mathrm{mg}$ pack (BNF 2011-12). The cost of Azithromycin is now £1.50. The most recent findings in practise have suggested that the clinical effectiveness of Doxycycline may be superior to Azithromycin.

Interpretation

In health economic terms, if Doxycycline is clinically superior and costs less than Azithromycin, then Doxycycline regimens dominate. This could have saved significant costs in 2011/12 (up to $£ 500,000$ ). Now the costs are broadly equivalent, providing that the cheaper form of Azithromycin is prescribed. Doxycycline is also a valid choice of therapy, and extended regimens of Azithromycin could now also be considered without being prohibitively expensive.

Policy recommendation

Within the context of treatment of asymptomatic, uncomplicated chlamydia diagnosed through screening, Azithromycin is likely to continue to be the drug of choice. The process for implementing changes to prescription should be made more streamlined and transparent, so that evidence can be more rapidly translated into cost-savings.

\section{P6.007 ADDRESSING ENDEMIC RATES OF STI IN REMOTE ABORIGINAL COMMUNITIES IN AUSTRALIA USING QUALITY IMPROVEMENT AS A KEY STRATEGY: THE STRIVE STUDY}

doi:10.1136/sextrans-2013-051184.1161

1,2J Ward, ${ }^{2} \mathrm{R}$ Guy, ${ }^{2} \mathrm{~L}$ Garton, ${ }^{3} \mathrm{~B}$ Silver, ${ }^{3} \mathrm{D}$ Taylor-Thomson, $4,2 \mathrm{~B}$ Hengel, ${ }^{2} \mathrm{~J}$ Knox, ${ }^{2} \mathrm{~S}$ McGregor, ${ }^{3,5} \mathrm{~A}$ Rumbold, ${ }^{2} \mathrm{~J}$ Kaldor. ${ }^{\prime} B a k e r$ IDI, Alice Springs, Northern Territory, Australia; ${ }^{2}$ The Kirby Institute, University of New South UK, Sydney, Australia; 'Menzies School of Health Research, Darwin, Northern Territory, Australia; ${ }^{4}$ Apunipima Cape York Health Council, Cairns, Queensland, Australia; ${ }^{5}$ University of Adelaide, Adelaide, South Australia, Australia

Background Remote Aboriginal communities in Australia have substantially higher rates of chlamydia (CT), gonorrhoea (NG) and trichomoniasis (TV) diagnosis compared with non-Indigenous people, despite many years of programme and policy aimed at reducing disparity.

Methods 'STRIVE' is a stepped wedge cluster randomised controlled trial, underway in 68 remote Aboriginal communities. The primary objective is to assess if a sexual health quality improvement programme within primary care clinics improves STI management and in turn reduces STI prevalence. Data is extracted from both patient management systems and from laboratories. To evaluate the impact of the trial, the largest STI prevalence study in Aboriginal people has been undertaken twice.

Results In the first year of the trial, testing rates have improved overall by $27 \%$ at 21 sites and by $50 \%$ or more at 11 sites. In 2011 , baseline prevalences for CT and NG $(n=2483)$ and TV $(n=1848)$ among 16-34 year olds were $9 \%, 7 \%$ and $13 \%$ respectively. Highest 